\title{
Testicular transcriptional signatures associated with high fertility
}

\author{
Marten Michaelis ${ }^{1, *}$, Alexander Sobczak1,*, Dirk Koczan², Martina Langhammer³, \\ Norbert Reinsch ${ }^{3}$, Jennifer Schön ${ }^{1}$ and Joachim M Weitzel ${ }^{1}$ \\ 'Institute of Reproductive Biology, Leibniz Institute for Farm Animal Biology (FBN), Dummerstorf, Germany, \\ ${ }^{2}$ Institute of Immunology, University of Rostock, Rostock, Germany and ${ }^{3}$ Institute of Genetics and Biometry, \\ Leibniz Institute for Farm Animal Biology (FBN), Dummerstorf, Germany \\ Correspondence should be addressed to J M Weitzel; Email: weitzel@fbn-dummerstorf.de or michaelis@fbn-dummerstorf.de \\ *(M Michaelis and A Sobczak contributed equally to this work)
}

\begin{abstract}
Factors of high fertility are poorly described. The majority of transgenic or knockout models with a reproductive phenotype are subfertile or infertile phenotypes. Few genotypes have been linked to improved reproductive performance $(0.2 \%)$ or increased litter size (1\%). In this study, we used a unique mouse model, fertility line FL1, selected for 'high fertility' for more than 170 generations. This strain has almost doubled the number of littermates as well as their total birth weight accompanied by an elevated ovulation rate and increased numbers of corpora lutea compared to a randomly mated and unselected control line (Ctrl). Here, we investigate whether the gonadal tissue of FL1 males are affected by 'co-evolution' after more than 40 years of female-focused selection. Using microarrays, we analysed the testicular transcriptome of the FL1 and Ctrl mice. These data were also compared with previously published female gonadal transcriptional alterations. We detected alterations in testicular gene expression, which are partly associated with female reproductive performance. Thus, female-focused selection for litter size has not only affected the female side, but also has been manifested in transcriptional alterations on the male gonadal organ. This suggests consequences for the entire mouse lines in the long run and emphasizes the perspective of inevitably considering both genders about mechanisms of high fertility. Reproduction (2018) 155 219-231
\end{abstract}

\section{Introduction}

Many genes necessary for reproductive performance are shared by both sexes. The similar gene expression patterns of ovary and testis are evidence of their close relationship as germinal organs. The Mouse Genome Informatics (MGI) gene expression database (www.informatics.jax.org) harbours mouse tissuespecific expression data. After extracting all annotated testicular and ovarian transcripts for co-expression analysis, we found that more than $50 \%$ of testicular expressed genes are also found in the ovary (Fig. 1, bright subsets). If considering only male and female gonadalspecific transcripts, the intersection between both reproductive organs is more than its divergence (Fig. 1, darkened subsets). A similar relationship between both gonadal tissues has been reported at the protein level (Kosti et al. 2016).

Given the complexity of the reproductive processes, the germinal organs are susceptible to alterations leading to reduced fertility or infertility (Evers 2002, Matzuk \& Lamb 2008, Devroey et al. 2009). As many cases of impaired fecundity remain idiopathic, it is important to identify animal models, particularly mouse models, with reproductive phenotypes, thereby facilitating investigations into fertility mechanisms. Indeed, more than 2000 mouse models with reproductive phenotypes can be found within the MGI databases. The majority of existing mouse models are transgenic or knockout mice exhibiting an infertile or subfertile phenotype (99\%). Our institute generated two unique outbred fertility mouse lines (named FL1 and FL2) that have been established by long-term selective breeding for high reproductive performance. The advantage of this approach is its heterogeneity, which more closely mimics the phenotypical alterations in humans than single gene approaches like classical transgenic or knockout models. Breeding strategies and comparative data for these high-fertility mouse lines have been described previously (Dietl et al. 2004, Langhammer et al. 2014, 2017).

Here we focus on the FL1 mouse line, which, after more than 170 generations of breeding, has almost double the selection traits litter size, and litter birth weight in comparison to the control line (Ctrl). This is accompanied by no sign of growth retardation in the 
Testicular transcripts

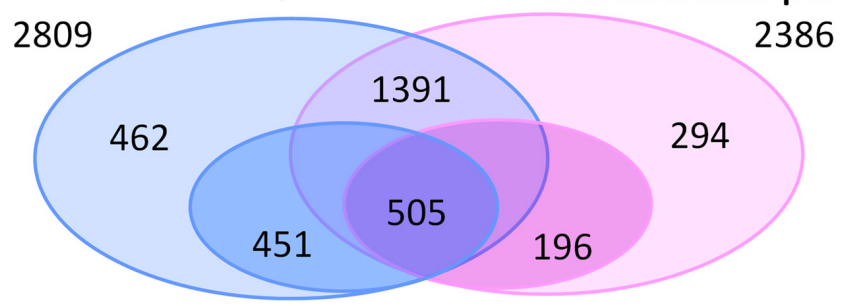

Figure 1 Genes expressed in male and female gonadal tissue. The number of genes expressed in postnatal testis (blue) and ovary (magenta) based on the MGI Gene Expression Database (www.informatics.jax.org/expression.shtml; extracted Jan 2017). Subsets of exclusively gonad-specific expressed genes are indicated by dark shading.

offspring litters and no alteration in pup survival after weaning (Langhammer et al. 2014).

The selection parameters of the FL1 line are primarily female-focused and are accompanied by physiological alterations within the ovary of FL1 dams (Spitschak et al. 2007). Previous work has shown that the ovary is the primary organ affected by this selection process. Females of the high-fertility line can ovulate more eggs and harbour almost twice as many corpora lutea $(\mathrm{CL})$ as the Ctrl mice (Spitschak et al. 2007). Furthermore, FL1 females generally have an imbalance between the number of ovulated eggs and CL. In fact, FL1 females are prone to develop a considerable number of polyovulatory follicles (POF), harbouring up to seven oocytes per follicle (Alm et al. 2010). In addition, alterations have been detected at the global ovarian transcriptional level (Vanselow et al. 2008).

On the male side, initial characterisations of FL1 bucks reveal altered sperm motility characteristics, as well as elevated lifetime expectancy and increased explorative phenotype. Furthermore, we detected changes in testicular maker genes pointing to alterations not only on the female side but the male side as well (Michaelis et al. 2013, Langhammer et al. 2014). Therefore, we hypothesize that after 40 years of femalefocused selection, the male germinal organ of FL1 mice has also been affected, due to the ovarian adaptation of highly increased ovulation rates.

Here, we examine the germinal organ transcriptome of male FL1 mice and make comparison to previously published transcriptomic data from FL1 ovaries (Vanselow et al. 2008). These data are informative about the extent of gender-focused fecundity selection.

\section{Materials and methods}

\section{Animal model and ethics statement}

The animal experiments were performed following national and international guidelines and were approved by the local authorities (Landesamt für Landwirtschaft,
Lebensmittelsicherheit und Fischerei, MecklenburgVorpommern, Germany).

Up to the 162 nd generation, the mice were kept in a semibarrier unit. After relocation into a newly built animal facility, the mice were maintained under a specific pathogen-free (SPF) environment with a 12-:12-h light-darkness (cycle) regime and ad libitum access to water and food (ssniff M-Z, Soest, Germany) at the Leibniz Institute for Farm Animal Biology, Dummerstorf (FBN).

The FL1 and Ctrl mouse lines were originally derived from the same genetic pool of a mixture of eight defined founder mouse lines (Dietl et al. 2004). The FL1 fertility line was selected over a long-term breeding process for (i) number of offspring and (ii) total litter birth weight. These criteria were combined into a fertility breeding index $=1.6 \times$ litter size + litter weight (at first delivery). Using the selection index for ranging the females offspring of the largest and heaviest litters were recruited for breeding of the next generation (Schüler L 1982). After 162 generations of index selection, breeding procedure was replaced by BLUP (Best Linear Unbiased Prediction) breeding value estimation, focused only on the number of newborn pups per litter in primiparous females (Langhammer et al. 2017). The population size of this outbred model has been maintained at 60-100 mating pairs per generation, ensuring its heterogeneous character. The unselected control line (Ctrl) has been sustained by using a rotational mating scheme with 125-200 breeding pairs per generation and avoiding full-sib mating. Hence, this outbred mouse line could preserve its properties over generations to ensure a significantly lower inbreeding coefficient compared to common inbreeding lines (Langhammer et al. 2017). Details regarding breeding proceedings can be found in Dietl and coworkers, Schüler and coworkers and Langhammer and coworkers (Schüler L 1982, Dietl et al. 2004, Langhammer et al. 2017). The males of this investigation have been further described in a two-factorial breeding experiment to delineate the impact of males and females on fertility parameters (Langhammer et al. 2017).

\section{RNA extraction}

Testicular RNA was extracted of 12- to 13-week-old males. For the transcriptional analysis, we used 16 mice per line, subdivided into two separate sets of biological replicates (each of eight animals per set and line). Mice were killed by $\mathrm{CO}_{2}$ inhalation, and the testes were dissected to be either snap frozen in liquid $\mathrm{N}_{2}$ or preserved in RNAlater (Ambion). Tissue disruption was done in RLT buffer (RNeasy kit, Qiagen) using a FastPrep-24 device (MP Biomedicals, Santa Ana, CA, USA) and Lysing Matrix D disposable tubes. RNA was extracted by the phenol/chloroform method, followed by spin column chromatography using the RNeasy Kit (Qiagen) protocol according to the manufacturer's instructions.

In the case of snap-frozen tissue the InviTrap Spin RNA Mini Kit (Stratec, Birkenfeld, Germany) was used for whole RNA extraction. The quantification was done spectrophotometrically using a NanoDrop 1000 device (Thermo Fisher Scientific), and the RNA integrity was checked by capillary electrophoresis (Agilent 2100 Bioanalyzer). 


\section{Microarray experiment}

For transcriptome profiling, we used the GeneChip Mouse Transcriptome Array (MTA) 1.0 (released in May 2015; currently termed Clariom D assay) by Affymetrix. RNA labelling and hybridization were executed at the Core Facility for Microarray Analysis, University of Rostock. Briefly, as recommended, $200 \mathrm{ng}$ of quality controlled total RNA was used for cDNA preparation and labelled with the GeneChip WT PLUS Reagent Kit (Affymetrix). The fragmented ( 100 nucleotides) and biotinylated ssDNA was hybridized for $16 \mathrm{~h}$ at $45^{\circ} \mathrm{C}$ to Affymetrix Gene Chip MTA 1.0, followed by washing and staining steps, using the Affymetrix Fluidics Station 450 according to standard protocols. Chips were then scanned at 0.7-micron resolution using the GeneChip Scanner 3000 7G (Affymetrix). The resulting image files (.DAT) were processed using Affymetrix GeneChip Command Console 4.0 (AGCC 4.0.0.1567) software for generating cell intensity files (.CEL). Raw data cell intensity files (.CEL) were normalized by the Robust Multiarray Average (RMA) algorithm with a Signal Space Transformation (SST) employing the Affymetrix Expression Console software (EC, version 1.4.1.46). All hybridizations were assessed for quality requirements, and raw data were submitted to the Gene Expression Omnibus (GEO) database according to MIAME guideline (GSE86063).

\section{Microarray experimental design}

Our microarray experimental design comprised two separated sets of microarrays. Initially, testicular RNA of eight animals per line was hybridized to eight individual microarrays with the intention to analyse for differentially expressed genes (DEGs) (FL1 vs Ctrl), employing fold change (FC) and statistical filter criteria. The resulting DEG lists were experimentally verified by a second microarray set. For revaluation, we used the same group size of eight animals per line as independent biological replicates pooled in equivalent amounts and hybridized to one single 'pool microarray' for each mouse line.

Using this approach, we ensured a comprehensive verification of the entirety of gained gene expression results of the first round of microarray hybridizations. This allowed us to exclude 'false-positive' transcripts, which were initially indicated as differentially expressed but could not be confirmed in independent re-evaluation experiments. This experimental approach has been applied to the second mouse line (FL2) and is currently submitted to another journal in a parallel manuscript.

\section{Quantitative real-time PCR}

Microarray results were additionally confirmed for selected genes by quantitative real-time PCR (qPCR). To this end, $0.5 \mu \mathrm{g}$ of total RNA was reverse transcribed using random hexamer primers and the iScript cDNA Synthesis kit (BioRad), in accordance to the manufacturer's instructions. For qPCR, $2 \mu \mathrm{L}$ of a 1:5 dilution of each cDNA was amplified with technical duplicates using SYBER Green mix (Bio-Rad), as previously described (Michaelis et al. 2013). The cycling conditions for the PCR procedure were identical (40 cycles of $30 \mathrm{~s}$ at $95^{\circ} \mathrm{C}, 45 \mathrm{~s}$ at $56^{\circ} \mathrm{C}$, and $30 \mathrm{~s}$ at $72^{\circ} \mathrm{C}$ ). Primers were designed using Primer-BLAST (http://www.ncbi.nlm.nih.gov/ tools/primer-blast) (Ye et al. 2012). If possible, special care has been taken to select oligonucleotides binding to intronoverspanning exons. Also, specificity was controlled by gel electrophoresis. The primers are listed in the Supplementary Table 1 (see section on supplementary data given at the end of this article). Samples were normalized to a combination of reference genes $(36 B 4, G A P D H, H P R T, B 2 m)$ and statistically evaluated using the Relative Expression Software Tool (REST 2009) (Pfaffl et al. 2002).

\section{Hormone analysis}

Serum progesterone was measured by ${ }^{3} \mathrm{H}$-radioimmunoassay $\left({ }^{3} \mathrm{H}-\mathrm{RIA}\right)$ with $[1,2,6,7-3 \mathrm{H}]$ progesterone tracer (Hartmann Analytic, Germany), as previously described (Brussow et al. 2011 ). Briefly, $50 \mu \mathrm{L}$ of serum in duplicates ( $n=14$ per group) was analysed with an incubation step at $37^{\circ} \mathrm{C}$ for $30 \mathrm{~min}$ and at $4^{\circ} \mathrm{C}$ for $2 \mathrm{~h}$. The $\mathrm{B} / \mathrm{F}$ (bound to free antigen) separation was performed by the dextran-charcoal method. Counting of radioactivity was accomplished by a Liquid Scintillation Counter with an integrated RIA program (TriCarb 2900 TR; Perkin-Elmer). Intra-assay and interassay precision were estimated as $7.6 \%$ and $9.8 \%$ for progesterone, respectively. The standard curve ranged from 62.5 to $16,000 \mathrm{pg} / \mathrm{mL}$ and the detection limit was $70 \mathrm{pg} / \mathrm{mL}$. The Student's $t$-test was used for statistical analyses.

\section{Availability of data}

Microarray data were submitted to the NCBI Gene Expression Omnibus (GEO) under accession number (GEO: GSE86063).

\section{Ethics approval}

The animal experiments were approved by the local authorities (Landesamt für Landwirtschaft, Lebensmittelsicherheit und Fischerei, Mecklenburg-Vorpommern, Germany).

\section{Results}

\section{Animal model}

FL1 mice were selected for a high number of offspring and increased total litter weight at birth via an established breeding index combining both selection criteria (breeding index $=1.6 \times$ litter size + litter weight). The factor 1.6 reflects the average birth weight of single mouse pups at birth and is applied to avoid growth retardation in the offspring population. Thus, only animals of the largest and heaviest litters were recruited for further breeding.

Due to the lack of selection pressure, the breeding parameters for Ctrl remained the same over the generations (Fig. 2A and Langhammer et al. 2014). After 172 generations, the Ctrl strain had a litter size of $11.4 \pm 3.3$ (mean \pm S.D.) and a total birth weight of $20.8 \pm 4.8 \mathrm{~g}$ (mean \pm S.D.). These data are similar 


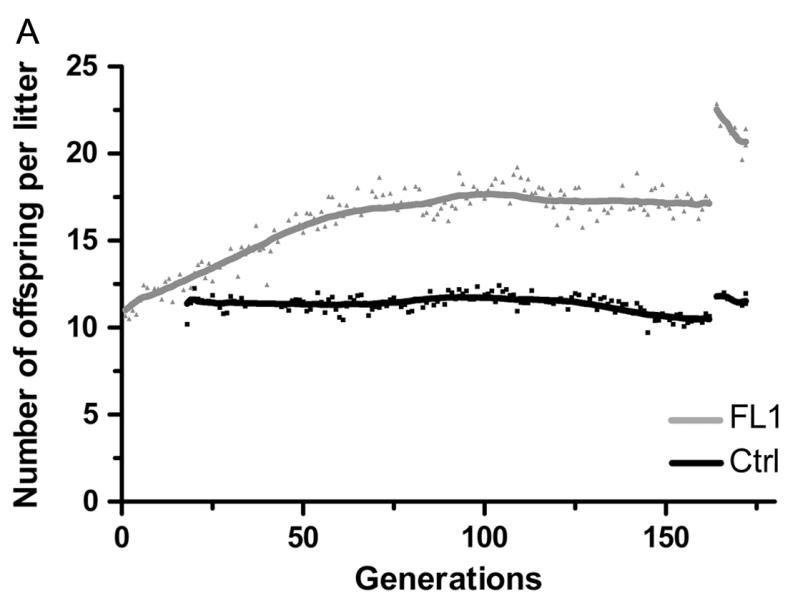

B
\begin{tabular}{|l|c|c|}
\hline Mouse line & Ctrl & FL1 \\
\hline Generations & 172 & 173 \\
\hline Offspring per litter & $11.4 \pm 3.3$ & $20.5^{* * *} \pm 4.0$ \\
\hline Birth weight per litter [g] & $20.8 \pm 4.8$ & $37.5^{* * *} \pm 6.7$ \\
\hline
\end{tabular}

Figure 2 Breeding success (number of offspring per litter) over the generations (A). From generation 163 onwards, all mouse lines have been selected and maintained in a new animal facility. Presumably, due to the improved housing conditions (specific pathogen-free, SPF), there was an increase in the selection criteria 'litter size' in all mouse lines (see a shift in offspring per litter from the 162 nd to $163 \mathrm{rd}$ generation). Data until generation 161 have already been presented in the study by Langhammer and coworkers (Langhammer et al. 2014). The number of offspring per litter and total litter weight at birth (mean \pm S.D.) for Ctrl and FL1 after $>170$ generations of selection to $\mathrm{Ctrl}$ are indicated (B). ${ }^{* * *}$ Will indicate statistically significant differences between FL1 and Ctrl ( $t$-test, $P<0.001)$.

to published data for other unselected mouse lines (Bowman \& Falconer 1960, Holt et al. 2004, Suto 2015). In contrast, the female-focused selection pressure for larger and heavier litters continuously increased the FL1 litter size over the generations (Fig. 2A). From generation 162 to 163 , the mouse lines had been moved to a new mouse facility. Presumably, due to the improved housing conditions (SPF), all mouse lines profited of the transfer (Fig. 2A, shift in offspring per litter from generation 162 to 163 ). After 173 generations, the selected traits were increased by $180 \%$ in FL1 line compared to the Ctrl line. FL1 now produce $20.5 \pm 4.0$ offspring and a total birth weight of $37.5 \pm 6.7 \mathrm{~g}$ per litter (Fig. 2B). These distinct line differences for both breeding values are significant $(P<0.001)$. In previous publication, we reported on a weight increase of the mothers/females in the FL1 line, possibly in reflection of the increased gestational burden (Langhammer et al. 2014). As such, we analysed the reproductive selections traits relative to its female's body weight. However, when put in relation to the body weight, the litter size increase is $165 \%$ and the litter weight increase $168 \%$, in comparison to Ctrl females at the time of mating (Supplementary Fig. 1). The gain in reproductive performance in FL1 was not accompanied by body weight loss of individual FL1 offspring, due to the consideration of a birth weight trait in the selection index. Statistical data represent means \pm S.D. and have been analysed by unpaired $t$-test (group size: 120 (Ctrl); 55 (FL1)).

\section{Expression profiling}

Within the germinal organs, males and females share many genes essential for reproductive performance. We assume that alterations on a transcriptomic level have gradually established over the selection process for increased fertility performance, not only for the female (Vanselow et al. 2008) but also for the male reproductive organ. To test this notion on a molecular level, we employed whole transcriptome analysis using the GeneChip MTA 1.0 (Affymetrix) providing broad transcript coverage for more than 66,100 coding and non-coding probe sets (PS).

The overall cell intensity distribution for each microarray before and after normalization (SST-RMA) is evidence for an overall well-performed hybridization experiment (Supplementary Fig. 2). However, to evaluate the overall transcriptional difference of the two mouse lines (FL1 vs Ctrl) and to demonstrate any potential effects in the males, a principal component analysis (PCA) was carried out. This was done in an unbiased manner, based on the 1st microarray set with the individual animal hybridizations, to determine whether FL1 breeding effects are visible at the overall transcriptional level. Additionally, this approach allowed us to identify any potential unusual observations, which could then be investigated before application of a supervised method. The resulting plot has a clustering congruent with a line-based grouping and represents an overall PCA mapping of 53.8\% (Fig. 3A). The particular principal components are denoted.

To further explore the variability of the dataset, we performed a hierarchical cluster analysis of the 500 most diversely expressed genes. In line with PCA results, FL1 and Ctrl transcripts clustered corresponding to their distinctive populations (Fig. 3B). Additionally, the gene expression data are more homogenous for the FL1 bucks than the Ctrl males, possibly due to the selection pressure applied to the high-fertility line. Contrarily, the randomly selected Ctrl line exhibits a more heterogenic expressed transcriptional profile.

To display the experimental benefit of the 'two array sets approach', we used nonparametric Spearman correlation analysis in dependence of varyingly stringent filter proceedings for the two array sets, plotting the FC of each transcriptome data set (Fig. 4). For the unfiltered 65770 probe set (PS), the correlation between the FCs of the 1st and 2nd array sets was assessed as 0.2628 (Fig. 4B). After increasing the stringency by 


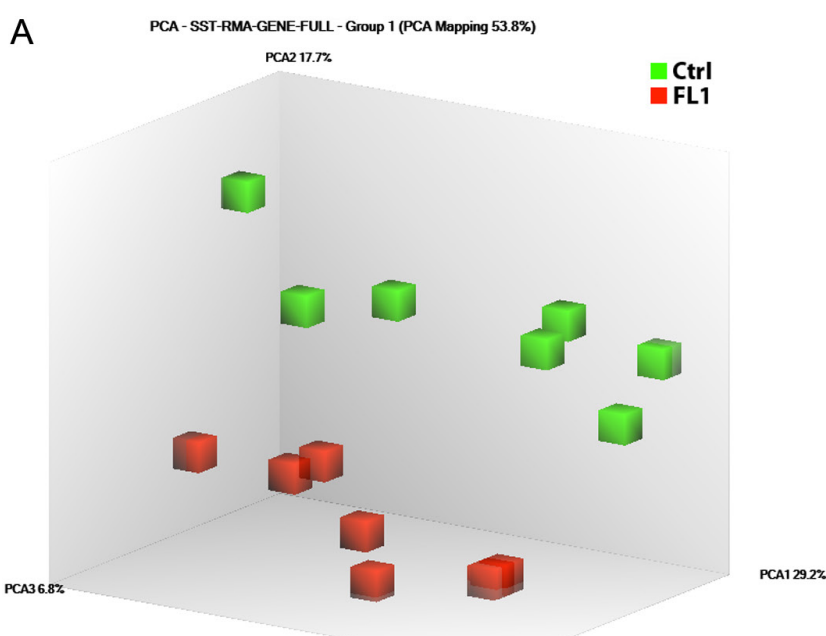

B

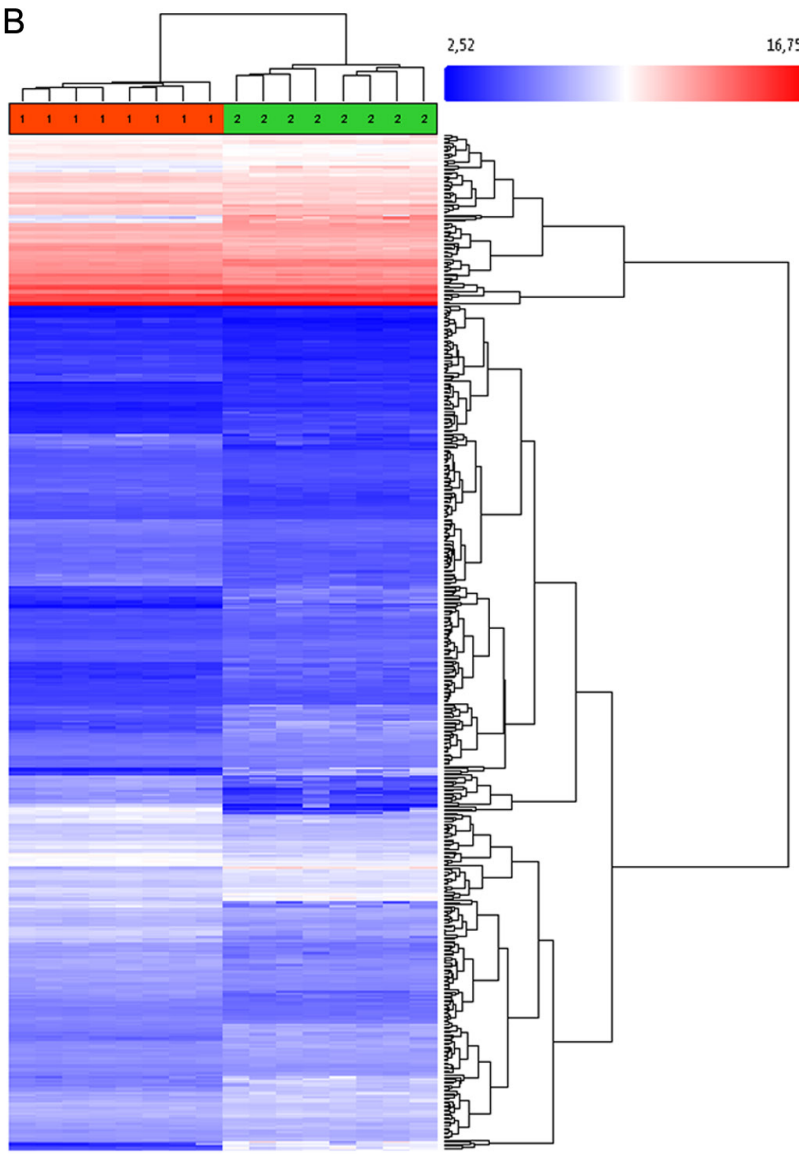

Figure 3 Principal component analysis (PCA) plot (A) of the individual testicular transcriptional profile of enrolled samples. Each cube represents one single microarray of FL1 (red) and Ctrl (green) mice. The PCA plot was generated using Expression Console software (Affymetrix). Hierarchical cluster analysis (B) was performed unsupervised for the 500 most DEGs in all groups. Genes are indicated vertically; the individual testicular samples are designated horizontally (FL1 red; Ctrl green). The intensity of red or blue colour suggests signal intensity as annotated by the scale.

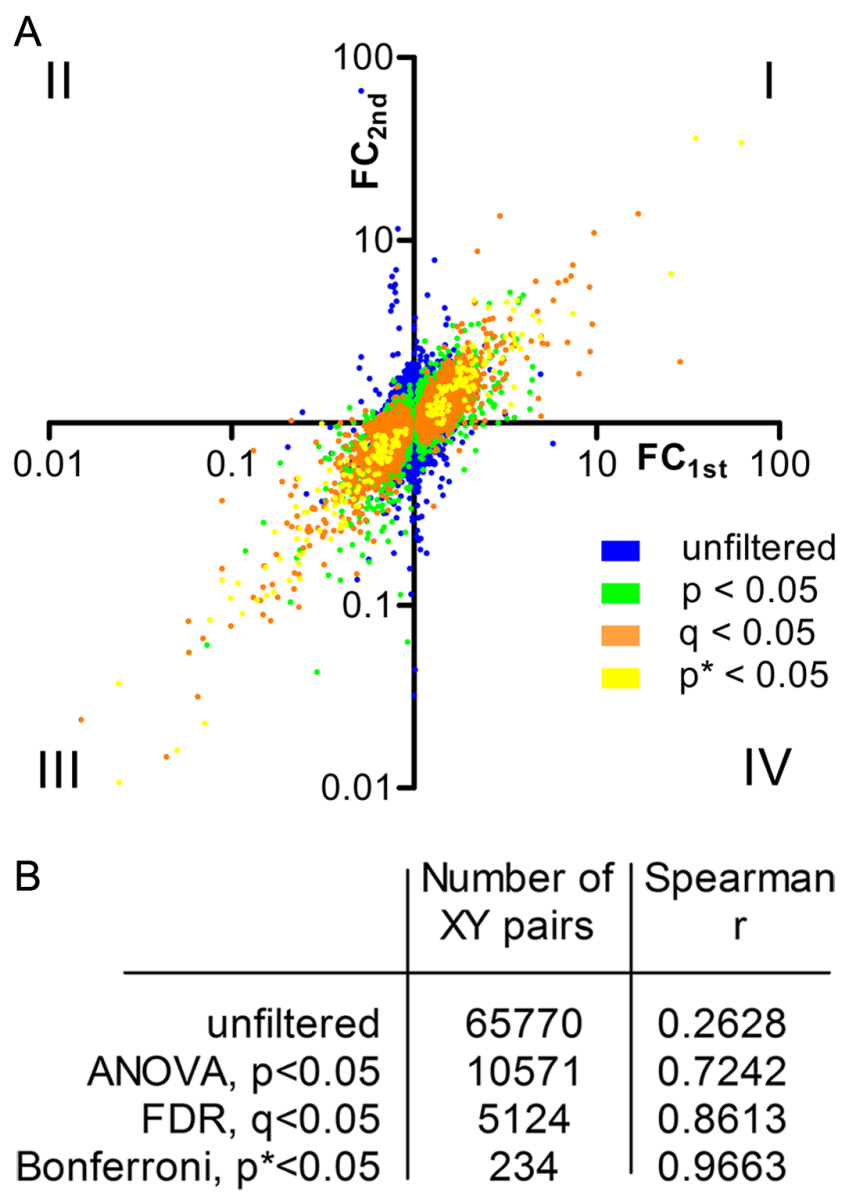

Figure 4 Scatter plots showing the correlation between the two sets of microarray experiments. Abscissae indicates FCs of the 1st microarray experiment. Ordinates represent FCs of the 2nd array validation experiment. Colour code indicates the dependence upon statistical filtering based on the 1st array set that enrolled animal individual hybridizations (blue: unfiltered; green: ANOVA, $P<0.05$; orange: FDR, $q<0.05$; yellow: Bonferroni correction, $\left.P^{*}<0.05\right)(\mathrm{A})$.

Correlation coefficient according to Spearman $(r)$ depending on the significance filtering and the number of enrolled $\mathrm{XY}$ pairs (probe set pairs) (B).

plotting only FCs that passed a statistical filter (such as ANOVA $(P<0.05)$, false discovery rate (FDR; $q<0.05)$ or Bonferroni correction $\left(P^{*}<0.05\right)$ based on the $1 \mathrm{st}$ array set), the correlation coefficient increased from 0.7242 to 0.9663 , depending on the applied filter (Fig. 4B). However, depending on the filter method, the number of PS passing these statistical criteria decreased dramatically from 10571 using ANOVA $(P<0.05)$ to 234 applying the Bonferroni correction $\left(P^{*}<0.05\right)$. Thus, PSs in quadrant II and IV (Fig. 4A) are supposed to be 'falsepositive' DEGs due to a lack of biological validation of its expression intensity and direction as seen in the $1 \mathrm{st}$ array set. Therefore, without the information of the 2 nd microarray set, it would be impossible to filter out these 'false-positive' transcripts. 
Each tissue expresses a subset of genes. To identify only transcripts that were present in the tissue, we filtered for detection of gene expression. With the current generation of Affymetrix gene expression arrays, the probes are designed to cover the entire transcript ranging from $5^{\prime}$ to $3^{\prime}$-end, resulting in a PS. To classify a transcript/PS as being present, at least in $50 \%$ of the samples, the probes have to be detected above background (DABG) levels. For this analysis, we used the Transcriptome Analysis Console (TAC) software as recommended by Affymetrix (see TAC user manual chapter 'Identifying and validating alternative splicing events'). This filter for expression detection was employed in both microarray sets (see experimental design).

Out of the list of 65770 PSs, for FL1, 57227 PS (87\%) were classed as present. For the Ctrl strain, we detected 57851 PS (88\%)-expressed transcripts. These lists were considered as testis-expressed genes and used for the identification of DEGs.

To identify DEGs, we used the following criteria: (i) transcripts assigned as upregulated must have been expressed in the FL1 group; transcripts assigned as downregulated must have been detected in the Ctrl group; (ii) $\geq 1.5$ or $\leq-1.5$ FCs were set as cut-offs and (iii) DEGs had to passed statistical filter as indicated (ANOVA $(P<0.05)$, FDR $(q<0.05)$ or Bonferroni correction $\left(P^{*}<0.05\right)$, of the 1 st array set, as indicated).

To correct for multiple testing, it is recommended to correct for the tested null hypotheses. For microarray experiments, a common statistical algorithm is the concept of the FDR or the Bonferroni correction. However, both statistical algorithms are incapable to reflect the biological diversity. Figure 5B illustrates the validated DEG intersection or 'recovery rates' for both array sets after the consecutive performance of our filtering criteria. Using ANOVA or FDR as a filter, we detected moderate 'recovery rates' of $61 \%$ (PS: 739/1204) and 68\% (PS: $558 / 825)$ respectively. Only the Bonferroni correction resulted in a good correlation $89 \%$ (PS: 148/167) between the $1 \mathrm{st}$ and 2 nd array set, but this was also associated with a considerable reduction in the number of DEGs.

Using ANOVA in combination with our experimental validation method, we avoid the need for multipletesting corrections, 1204 PSs of the 1st array met the filter criteria for differential expression and had a $P$ $<0.05$ (ANOVA). Of these, 739 PSs could be validated by the 2 nd array set of biological replicates (Fig. 5B).

\section{Bioinformatics evaluation}

To detect the activation or inhibition of potentially relevant cellular or biological processes, gene ontology (GO) classification was performed for all differential expressed transcripts in dependence of upregulation or downregulation. However, of the 739 differentially expressed PS, almost half (375) are not annotated with official gene symbols or public gene IDs due to missing database information, resulting in lost resource of those

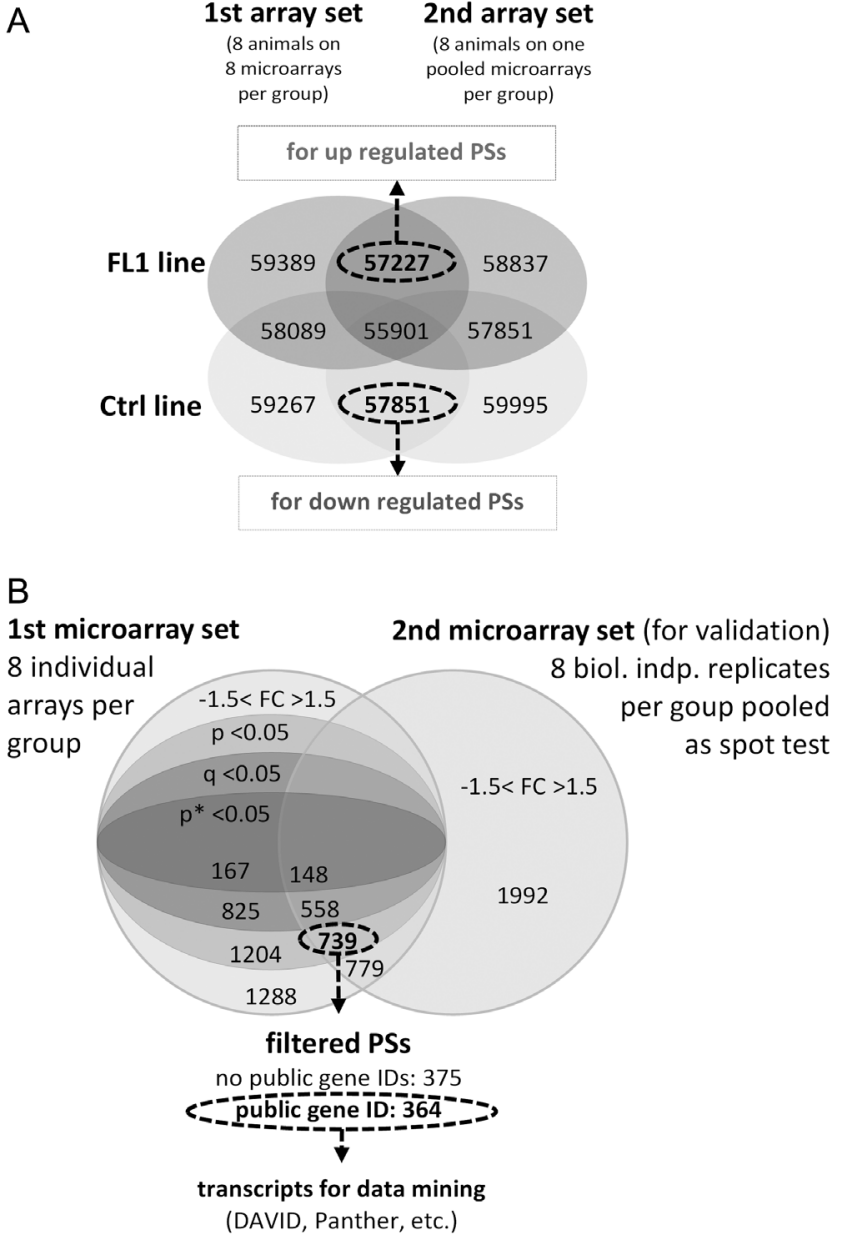

Figure 5 Microarray filtering strategy for the detection of differentially expressed genes (DEGs). For global gene expression analysis, a gene chip MTA 1.0 (Affymetrix) was used, equipped with 65770 probe sets (PS). The chip experiment was based on two biologically independent sample sets (1st, 2 nd array set) of two outbred mouse lines FL1 and Ctrl. The 1 st array set was analysed for differential gene expression while the 2 nd array set experimentally confirmed the findings of the 1 st set (see microarray experimental design of the methods section). Gene expression detection identified the number of actually expressed genes within each sample set and subsequently served as a first filter criterion. (A) The Venn diagram illustrates the number of differentially expressed genes (FL1 vs Ctrl) found in both 1st and 2nd microarray set $(\mathrm{FC}>1.5$ or $\mathrm{FC}<-1.5)$. (B) This Venn diagram represents the dependency of the verified DEG amount on the deployed statistical filter for the 1 st array set (ANOVA, $P<0.05$; FDR, $q<0.05$; Bonferroni correction, $\left.P^{*}<0.05\right)$.

mainly non-coding transcripts (Fig. 5B, see filtered PS). For rough classification, we identified 496 non-coding transcripts, of those: 314 lincRNAs, 124 antisense RNAs, 16 sense no-exonic RNAs and two microRNAs. However, the expression site is known for 105 non-coding RNAs, $70 \%$ (74 transcripts) are annotated as expressed in the testis and 52\% (55 transcripts) are exclusively expressed in the testis while $17 \%$ non-coding RNAs (18 transcripts) are related to oocyte expression site (Supplementary Fig. 3). The list of those DEGs with official gene 
symbols is provided in the Supplementary Table 2. GO classifications were able to identify 190 annotated mouse genes (www.geneontology.org) (Harris et al. 2004, Mi et al. 2005). The overall GO classification is shown in Fig. 6. FL1 transcripts associated with nucleic acid-binding properties were twice as likely to be downregulated as upregulated, whereas the reverse was true for transcripts with protein-binding properties.

Gene set enrichment analysis (GSEA) was used to evaluate functional differences between the FL1 and Ctrl testicular transcriptomes. Before this, duplicates within the gene list were excluded. The resulting list of 358 DEGs with official genelDs was subjected to the Database for Annotation, Visualization and Integrated Discovery (DAVID, david.ncifcrf.gov). We found 324 matches for murine annotations within the database. An extract of the GSEA with corresponding genes is presented in Table 1.

In general, the GSEA identified a wide range of biological as well as molecular categories that have been altered in FL1 bucks testis during the long-term breeding process. Including, enrichment of peptidase inhibitor activity, mainly of serine protease inhibitors and Serpin transcripts, half of which were upregulated (e.g. Serpine3, Serpini1, Serpini2) and half decreased expressed (e.g. Agt, Serpinb10, Serpina3a). In addition to those transcripts involved in proteolytic processes, we detected genes of the steroid hormone biosynthesis cascade and oestrogen metabolic processes to be downregulated in FL1 testis (e.g. Hsd3b1, Cyp1b1, Sult1e1). Besides, we noticed unexpected enrichments like adaptive immune response as well as haptoglobin-haemoglobin complex.

FL1 mouse line has been selected for increased reproductive performance. Based on that assumption and to detect fertility relevant alterations in the testicular transcriptome, we analysed our DEG lists for matches of mammalian phenotype (MP) with reproductive system phenotypes taking both genders into count, using the MGl database (www.informatics.jax.org). By this approach, we identified 12 mouse reproductive phenotype-associated genes that matched our DEG list. These genes are summarized in Table 2, with a selection of the most pronounced reproductive phenotypes.

The lists include the genes Agt and Ccnd2, which are known to be important for female fecundity and proper ovulation. While Agt expression is decreased, $\mathrm{C} C n d 2$ is upregulated in FL1 testis. In addition, $\mathrm{Nr} 2 \mathrm{c} 2$, associated with oligozoospermia, and Sult1e1, related to abnormal sperm motility, were downregulated in FL1 bucks.

\section{FL1 signatures of differential expression in male and female gonads}

High-fertility mouse line FL1 have been selected for increased reproductive performance via female index traits. Previous FL1 examinations focused on the dams, revealing an increase in ovulation rates due to increased

\section{GO terms - molecular function}

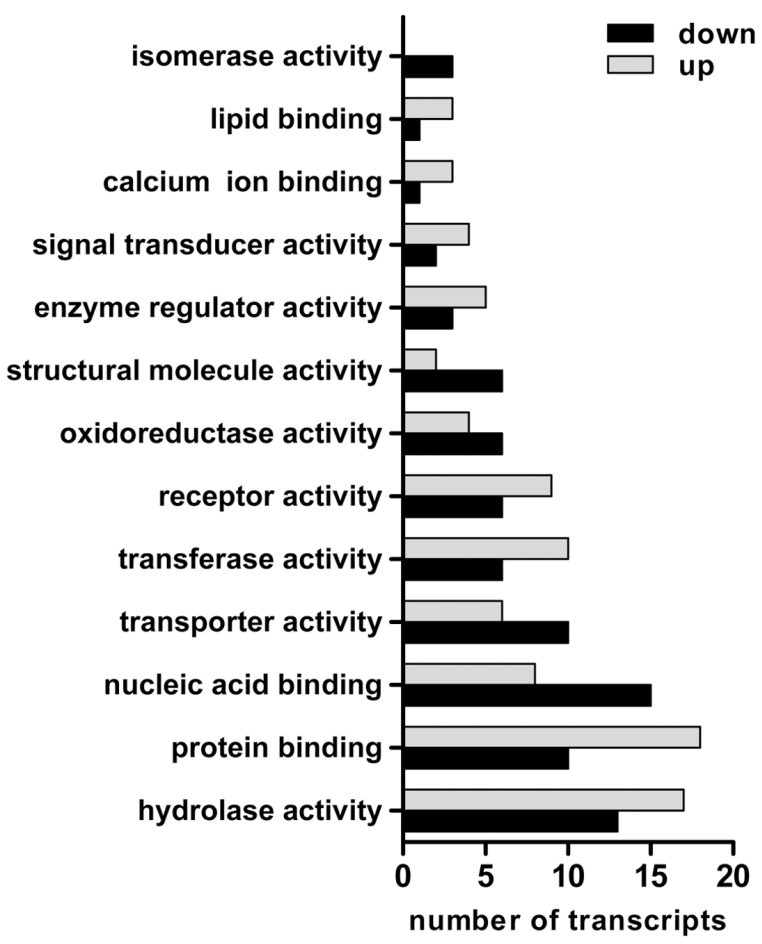

\section{B GO terms - biological processes}

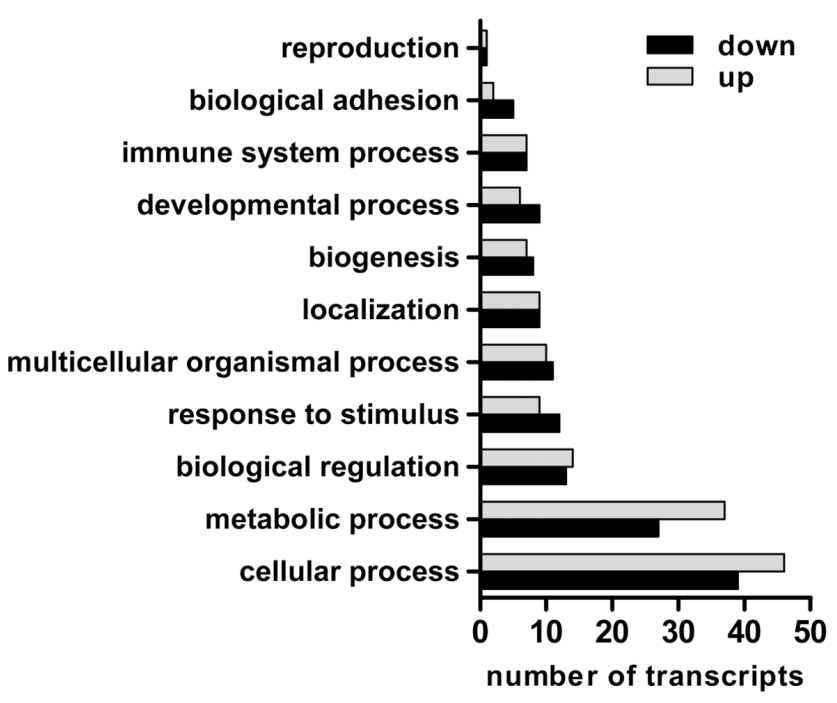

Figure 6 Gene ontology (GO) findings: Number of upregulated (red) and downregulated (blue) transcripts, as well as their assigned molecular functions (A) and biological processes (B).

oogenesis yields, which are partly reflected by POF (Spitschak et al. 2007, Alm et al. 2010). Consequently, the FL1 dams ovarian transcriptome was dissected, leading to the detection of gene expression alterations, which possibly could cause these extraordinary oocyte numbers as basis for the FL1 litter sizes. Based on 
Table 1 Gene set enrichment analysis (GSEA) for testis of FL1 vs Ctrl line using the platform Database for Annotation, Visualization and Integrated Discovery (DAVID).

\begin{tabular}{|c|c|c|c|}
\hline Term & Genes & Fold enrichment & Fisher $\boldsymbol{P}$-value \\
\hline Peptidase inhibitor activity & $\begin{array}{l}\text { CST9, WFDC10, SERPINB10, SERPINI1, } \\
\text { SERPINA3A, SERPINI2 }\end{array}$ & 5.38 & 0.005 \\
\hline Serpin & $\begin{array}{l}\text { AGT, SERPINE3, SERPINB10, SERPINI1, } \\
\text { SERPINA3A, SERPINI2 }\end{array}$ & 7.35 & 0.0005 \\
\hline Steroid hormone biosynthesis & HSD3B1, CYP1B1, SULT1E1 & 9.19 & 0.041 \\
\hline Oestrogen metabolic process & CYP1B1, SULT1E1 & 51.28 & 0.038 \\
\hline Steroid binding & SHBG, RXRA, SULT1E1, NR2C2 & 11.68 & 0.005 \\
\hline Steroid hormone mediated signalling pathway & RXRA, ABHD2, NR2C2 & 6.93 & 0.069 \\
\hline Haptoglobin-haemoglobin complex & HBA-A2, HBB-BS, HBB-BT & 61.24 & 0.001 \\
\hline Adaptive immune response & XRCC4, FAS, CD74, FCGR3 & 6.10 & 0.027 \\
\hline
\end{tabular}

The table represents an extraction of the functional analysis for overrepresented GO terms with indicated fold-enrichment score and Fisher Exact $P$ value.

these findings, we pursued a comparison of FL1 ovary and testis transcriptome variations towards Ctrl, to get information about gender-crossing FL1 transcriptional signatures of DEGs. These could apparently represent targets of influence by the long-lasting continuous breeding process and as such could be of high relevance for eliciting high fertility.

The considered ovarian microarray experiment was performed by Vanselow and coworkers (2008). Briefly, ovarian samples were originally collected at the

Table 2 List of genes associated with reproductive phenotypes found in the database Mouse Genome Informatics (MGI - www.informatics.jax. org) and corresponds to DEGs of FL1 compared to Ctrl.

\begin{tabular}{|c|c|c|c|c|}
\hline Gene symbol & FC 1st set & FC 2nd set & Term & MP ID \\
\hline Agt & $-2.6^{* * *}$ & -1.6 & Decreased ovulation rate & MP:0003355 \\
\hline Adamts 5 & $1.5^{* * *}$ & 1.6 & Reproductive system phenotype & MP:0005389 \\
\hline \multirow[t]{5}{*}{ Sult1e1 } & $-4.4^{* * *}$ & -3.2 & Abnormal sperm motility & MP:0002674 \\
\hline & & & Leydig cell hyperplasia & MP:0001152 \\
\hline & & & Abnormal seminiferous tubules & MP:0002216 \\
\hline & & & Reduced female fertility & MP:0001923 \\
\hline & & & Decreased litter size & MP:0001935 \\
\hline \multirow[t]{3}{*}{$\mathrm{Nr} 2 \mathrm{c} 2$} & $-1.7^{* * *}$ & -1.5 & Reduced male fertility & MP:0001922 \\
\hline & & & Oligozoospermia & MP:0002687 \\
\hline & & & Seminiferous tubule degeneration & MP:0001154 \\
\hline \multirow[t]{3}{*}{ Ccnd2 } & $2.2^{* *}$ & 2.1 & Abnormal ovulation & MP:0001928 \\
\hline & & & Abnormal ovarian folliculogenesis & MP:0001130 \\
\hline & & & Oligozoospermia & MP:0002687 \\
\hline \multirow[t]{5}{*}{ Cnbd2 } & $1.8^{* * *}$ & 1.6 & Male infertility & MP:0001925 \\
\hline & & & Reduced male fertility & MP:0001922 \\
\hline & & & Abnormal sperm motility & MP:0002674 \\
\hline & & & Azoospermia & MP:0005159 \\
\hline & & & Small seminiferous tubules & MP:0001153 \\
\hline \multirow[t]{5}{*}{ Ins/5/relaxin } & $-2.0^{*}$ & -3.3 & Male infertility & MP:0001925 \\
\hline & & & Female infertility & MP:0001926 \\
\hline & & & Decreased litter size & MP:0001935 \\
\hline & & & Abnormal estrous cycle & MP:0001927 \\
\hline & & & Asthenozoospermia & MP:0002675 \\
\hline \multirow[t]{4}{*}{ Nsun2 } & $-1.6^{* * *}$ & -1.9 & Male infertility & MP:0001925 \\
\hline & & & Oligozoospermia & MP:0002687 \\
\hline & & & Testis degeneration & MP:0013600 \\
\hline & & & Decreased circulating cholesterol & MP:0005179 \\
\hline \multirow[t]{4}{*}{$T t / / 1$} & $1.9^{* *}$ & 1.7 & Male infertility & MP:0001925 \\
\hline & & & Asthenozoospermia & MP:0002675 \\
\hline & & & Oligozoospermia & MP:0002687 \\
\hline & & & Abnormal spermatogenesis & MP:0001156 \\
\hline \multirow[t]{2}{*}{ Dnah11 } & $-2.2^{* *}$ & -1.7 & Reduced fertility & MP:0001921 \\
\hline & $*$ & & Decreased litter size & MP:0001935 \\
\hline Gpr133/Adgrd1 & $-2.7^{* *}$ & -2.4 & Female infertility & MP:0001926 \\
\hline \multirow[t]{2}{*}{ Mfge8 } & $2.1^{* * *}$ & 2.6 & Reduced male fertility & MP:0001922 \\
\hline & & & Decreased litter size & MP:0001935 \\
\hline
\end{tabular}

Genes, FCs for bot array sets, matching mammalian phenotype (MP) description and number are indicated.

FC of 1 st $(*, P<0.05 ; * *, P<0.01 ; * *, P<0.001)$ and 2 nd microarray set are indicated in table. 
metestrous stage and similarly prepared, filtered and processed like the investigated testis samples, differing in the employed microarray platform (see GSE11113). The ovaries were analysed by the previous 3' IVT (in vitro transcription) microarrays by Affymetrix being equipped with the feature of mismatched controlled expression detection. Those pervious microarrays consist of PS representing 11-20 probes targeting the 3' end of the mRNA (Della Beffa et al. 2008). As such the 3' IVT microarray, target more or less only transcripts that are protein-coding genes. However, our testicular transcriptional analyses are based on current microarray generation with PS ranging from $5^{\prime}$ to $3^{\prime}$-end. This makes it possible to analyse the transcriptional expression for splicing events. A list of alternative splicing events is provided in the Supplementary Table 3. However, we dismissed the feature to analysis for alternative splicing events and focused on differential gene expression to compare the testicular and ovarian transcriptional profiles. Furthermore, the 'new' exon gene chips are equipped with a wider range of transcripts, ranging from protein-coding to non-protein-coding RNAs (e.g. IncRNAs and microRNAs transcripts). Hence, the 'old' gene chips represent only a subset of transcripts found in the current array generation. As such, we only compared both germinal organs for coding transcripts.

For DEG comparison, we included the testis result tables derived from the two microarray set analyses and opposed them to the former DEG list of the FL1 ovary study summarized in Fig. 7A. We could reveal an intersection of 23 DEGs detectable in both FL1 testis and ovary when compared against Ctrl samples.
Among these, DEGs 15 are regulated in the same manner in the gonads of both genders, i.a. Agt, Sult1e1, Aldh1a7 and Serpina3a. In contrast, 8 transcripts are reversely expressed meaning sex-dependent observation of downregulation and upregulation for the same gene, e.g.: Cyp 1b1, Rd3 and Ccnd2.

Those transcriptional signatures we imported to the form FNTM (Functional Networks of Tissues in Mouse; fntm.princeton.edu) for network analysis. The inquiry was done for germ cells of both genders. For germ cells of the testis, there was a strong direct functional relationship between Agt, Aldh1a7, Atp6v0e2, Bdh1, Ccnd2, Cdk14, Cyp1b1, Dapp1, Fam132a, Figf, Fxyd6, H2-K1, Ifi204, Iqgap2 and Ptpn14, having Agt as a central linker (Fig. 7B). Unexpectedly, this strong network was not reflected in germ cells of the ovary. On the female side, we detected only moderate or weak functional relationships (Fig. 7C).

\section{Validation of microarray data with qPCR}

To validate our microarray data, we performed qPCR analysis of a selected set of transcripts intending to re-evaluate and to confirm the relative expression ratio of the 2 nd set microarrays. To overcome shortage of knowledge concerning individual expression variation of the 2 nd pooling array samples for qPCR. For this, we used the same mouse line testis RNA samples (before pooling) and analysed each sample's relative transcriptional abundance separately. Primers for qPCR were designed as oligonucleotides, binding to intronoverspanning exons if possible (list of primer sequences,
A

\begin{tabular}{|c|c|c|c|}
\hline \multicolumn{2}{|c|}{ Males } & \multirow{2}{*}{$\begin{array}{c}\text { Females } \\
\text { FC } \\
\text { array set }\end{array}$} & \multirow[b]{2}{*}{$\begin{array}{c}\text { Gene } \\
\text { Symbol }\end{array}$} \\
\hline $\begin{array}{c}\mathrm{FC} \\
1^{\text {st }} \text { array } \\
\text { set }\end{array}$ & 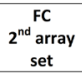 & & \\
\hline \multicolumn{4}{|c|}{ Similar differential expressed genes } \\
\hline $8.0^{* * *}$ & 1.9 & $1.9^{* *}$ & Dapp1 \\
\hline $2.5^{* * *}$ & 2.6 & $4.2^{* * *}$ & Fam132a \\
\hline $2.2^{* * *}$ & 2.2 & $1.7^{*}$ & Bdh1 \\
\hline $1.9^{* * *}$ & 2.5 & $3.0^{* * *}$ & Rnasel \\
\hline $1.6^{* * *}$ & 1.6 & $2.9^{* *}$ & Ifi204 \\
\hline $1.7 * * *$ & 2.0 & $1.7^{* * *}$ & Figf \\
\hline $1.7^{* * *}$ & 1.7 & $2.3^{* * *}$ & H2-K1 \\
\hline $1.7^{*}$ & 1.8 & $3.5^{* * * *}$ & Bhmt \\
\hline$-1.6^{*}$ & -1.8 & $-2.2 * * *$ & Serpina3a \\
\hline$-1.6^{* * *}$ & -1.6 & $-2.1^{* * *}$ & Iqgap2 \\
\hline$-2.1^{* *}$ & -3.1 & $-2.3^{* * *}$ & Aldh1a7 \\
\hline$-2.1 * *$ & -2.2 & $-2.7 * * *$ & Fxyd6 \\
\hline$-2.5^{* * *}$ & -2.2 & $-1.6^{*}$ & Cdk14 \\
\hline$-2.6^{* * *}$ & -1.6 & $-1.7^{* * *}$ & Agt \\
\hline$-4.4^{* * *}$ & -3.2 & -1.6 & Sult1e1 \\
\hline \multicolumn{4}{|c|}{ Opposite differential expressed genes } \\
\hline $9.7^{* * *}$ & 11.0 & $-1.8^{* *}$ & Rd3 \\
\hline $2.2^{* *}$ & 2.1 & $-2.2 * * *$ & Cond2 \\
\hline$-1.7^{* * *}$ & -1.8 & $1.5^{* * *}$ & Cyp1b1 \\
\hline$-1.9^{* * *}$ & -1.7 & $2.0^{* * *}$ & Pappa \\
\hline$-2.1^{* *}$ & -3.0 & $2.0^{* * *}$ & Atp6v0e2 \\
\hline$-2.4^{* * *}$ & -2.7 & $2.0^{* *}$ & Ptpn14 \\
\hline$-13.6^{* *}$ & -16.5 & $2.9^{*}$ & Ibsp \\
\hline$-17.2^{* * *}$ & -12.2 & 1.5 & Cst9 \\
\hline
\end{tabular}

B germ cell of testis

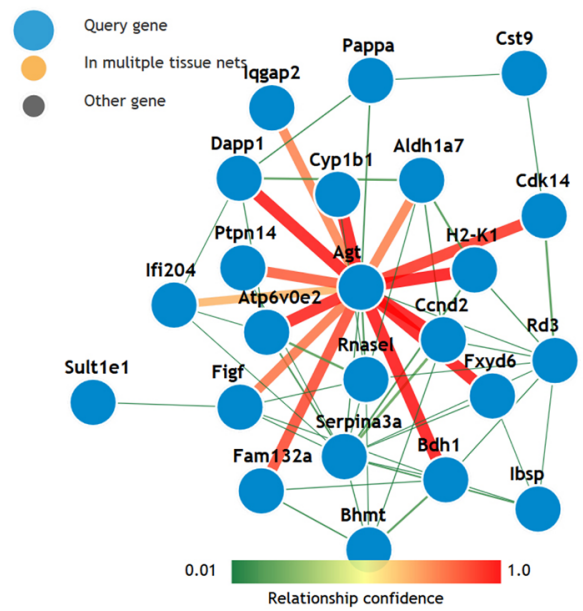

C germ cell of ovary

Figure 7 Testicular and ovarian differentially expressed genes, compared the FL1 to Ctrl strains (A). Testicular differential expression profile is based on two sets of microarray experiments ( 1 st and 2 nd array set; males). The ovarian transcriptional profile is based on previous work and has been extracted from the GEO database (GSE11113; females). Functional relation within testicular (B) and ovarian (C) germ cells of genes were found to be altered in high-fertile FL1 vs Ctrl mice, analysed by the Functional Networks of Tissues in Mouse platform (fntm.princeton.edu). Minimum (0.01, green) and maximum (1.0, red) relationship confidences are indicated by the colour code. 
Supplementary Table 1). All relative expression ratios were normalized to a combination of four reference genes (36B4, GAPDH, HPRT, B2m) using REST 2009 software (Pfaffl et al. 2002). Within this software, FC and $P$ value were generated for statistical evaluation.

By qPCR, comparing FL1 and Ctrl strains, we detected significant differences in expression of Sult1e1 (FC -3.0, $P<0.001$ ), CCnd2 (FC 1.8, $P<0.01$ ), Cdkl4 (FC $-2.4, P<0.001)$, Stat2 (FC $-4.1, P<0.001)$, Agt (FC $-1.8, P<0.001)$, Aldh1a7 (FC $-8.6, P<0.001)$, Cst9 (FC $-5.3, P<0.001)$ and Cyp1b1 (FC $-2.2, P<0.001)$. Hence, these specific 2nd array set qPCR transcription data revealed high similarity to both the 2 nd set array data itself (Fig. 8A, number below column) as well as to the biological-independent replicates of the 1 st array set (Fig. 8A, number in brackets below column). The overall agreement between the qPCR and microarray data was visualized using Spearman correlation (Fig. 8B). Except for Cdk/4, Aldh1a7 and Cst9, the correlation values demonstrated high similarities, even though qPCR and microarray represent two different techniques, which differ in mRNA detection target.

\section{Progesterone concentration in blood}

In a previous publication, we noticed an increase in serum corticosterone levels in FL1 males (Langhammer etal. 2017). Progesterone (P4) can serve as a precursor of the corticosteroid pathway. Furthermore, one of the key enzymes of P4 pathway is differentially expressed in FL1 testis on the transcriptional level. Hsd3b1 (FC: $-2.0^{* * *},-1.7 ; 1$ st and 2nd array set, respectively) encodes the enzyme $3 \beta$-hydroxysteroid dehydrogenase type 1, which is involved in steroid biosynthesis, converting pregnenolone to P4. Although this enzyme is associated with adrenal gland expression, according to the Biogps database, the ovary had the second highest abundance of $\mathrm{Hsd} 3 \mathrm{~b} 1$ mRNA (biogps.org/\#goto=genereport\&id=15492). In addition, we noticed high Hsd3b1 mRNA levels in the testis ((Ctrl: Avg Signal (log2): 10.18, 10.28 (1st, 2nd set); (FL1: Avg Signal (log2): 9.22, 9.53 (1st, 2nd set)). To test if differences in Hsd3b1 mRNA levels affect the hormonal concentration, the serum P4 level of FL1 and Ctrl males were examined by radioimmunoassay (RIA). To minimize the influence of circadian fluctuation all serum samples were collected at the same time of the day.

In Ctrl males, we measured $2.9 \pm 0.8 \mathrm{ng} / \mathrm{mL}$ total $\mathrm{P} 4$ from blood plasma samples (Fig. 9). This concentration was slightly higher than has been reported for C57BL/6 bucks (Schneider et al. 2003). Unexpectedly, compared to the Ctrl mice, the FL1 mice had significantly increased P4 values $(4.0 \pm 1.1 \mathrm{ng} / \mathrm{mL}, P<0.05, n=10$ animals per group). Statistical data are indicated as means \pm S.D. and have been analysed by unpaired $t$-test.
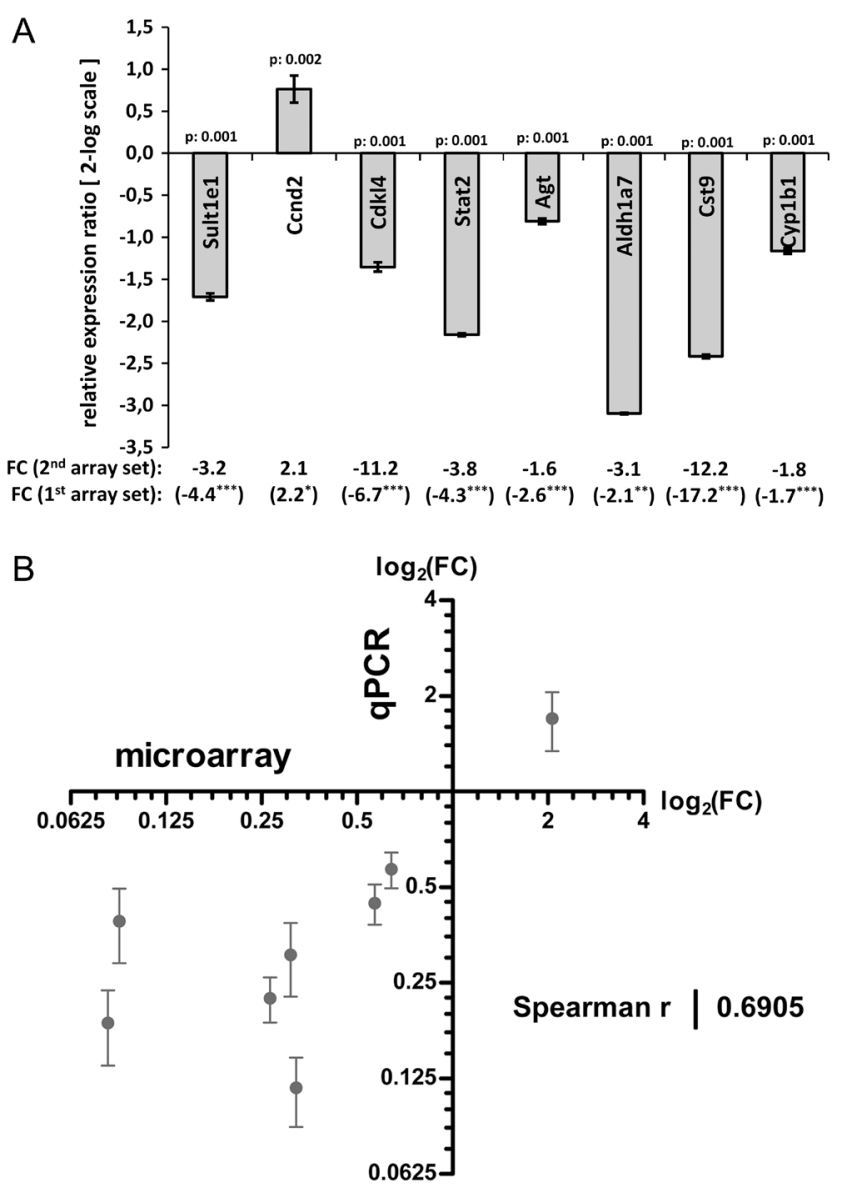

Figure 8 qPCR re-evaluation of selected, microarray-founded differentially expressed transcripts. Certain relative mRNA levels for 2 nd microarray set samples gained by using qPCR as an independent method are designated. Total testis RNA of individual FL1 and Ctrl mice was used prior to pooling for the 2 nd microarray set. Samples were normalized to a combination of reference genes (36B4, GAPDH, HPRT, B2m) and statistically evaluated employing the Relative Expression Software Tool (REST 2009) (Pfaffl et al. 2002). Relative expression ratios are presented as $\log 2$ bars and statistical significance is indicated above the columns (A). Numbers below bars indicate fold change (FC) values calculated for the 2 nd microarray set comprising the same testis samples as one pool. Numbers in brackets denote FCs of the 1 st microarray set as independent biological replicates. Scatter plot illustrates the correlation of 2 nd array set sample FCs acquired by both microarray (abscissae) and qPCR (ordinate), exposing the verification of expression data valid for specified transcripts (B).

\section{Discussion}

Here, we aimed to analyse the long-term effects and induced alterations in the males after more than 170 generations of female-focused high-fertility breeding. FL1 mouse line is generally distinguished by almost doubling the number of pups per litter as well as total litter weight, which can be explained by increased ovulation rate as has been previously reported (Spitschak et al. 2007). Initial studies regarding reproductive phenotype and characterisations of FL1 


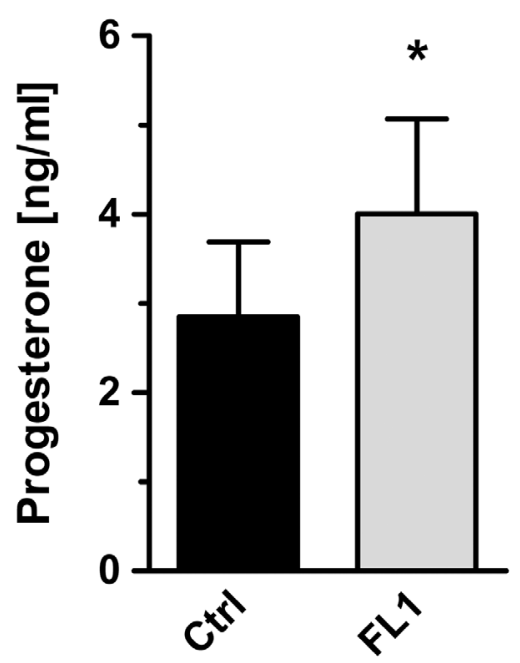

Figure 9 Progesterone. Plasma progesterone concentrations of FL1 and Ctrl males were analysed by radioimmunoassay (RIA). Individual values, mean and S.D. are shown. Groups were tested for normal distribution and analysed by two-tailed $t$-test $(* P<0.05)$.

males have shown alterations in testis composition, endocrinology and exploration behaviour compared to the Ctrl strain (Michaelis et al. 2013, Langhammer et al. 2014). The current study investigates the possibility of testis transcriptome alterations in the FL1 mice.

Using a microarray approach, we searched for changes in the testicular gene expression patterns that might have been engraved in the males by the long-term selection for increased female reproductive performance, based on the assumption that males and females share many genes essential for reproductive performance within their germinal organs. In addition, our institute already reported that, the selection for evermore larger and heavier litters is accompanied by raised ovulation rate and elevated numbers of CLs of the females (Spitschak et al. 2007). As such, some of the DEGs might be directly causative of the female-focused breeding process, whereas expression alterations observed in the fertility line testis might be induced as 'secondary' effect. Thus, we have to discuss transcriptional alterations in the light of male as well as female reproduction.

Mapping of genes to GO terms was used to detect overall biological processes or molecular functions that might be altered in FL1 mouse testis due to the longterm high-fertility selection process towards elevated litter sizes. Surprisingly, only two genes are associated with reproductive phenotypes, out of the 190 DEGs annotated in the database.

By functional annotation clustering analysis, we detected an enrichment for genes linked to peptidase inhibitory processes, mostly of the Serpin family. However, the physiological function of serpins within the testis is not well elucidated. One of the serpins found to be differentially expressed is Agt. This serpin is one of the major players in the renin-angiotensinogen system (RAS) and acts as a precursor of the active angiotensin (Ang) II within the angiotensin cascade. This hormonal system is known for its blood pressure regulation (Williams 2016). Also, there is increasing evidence for the local synthesis of RAS components in various tissues (Bader \& Ganten 2008), as well as for the direct action of angiotensin (Ang) II in male reproductive organs (Maruta \& Arakawa 1983). Nevertheless, the role of angiotensin as an effector in the testis is only partially understood. Besides its possible implication in male reproduction, Agt is of vital importance for ovarian function. In our work, Agt was found to be altered in FL1 testis as well as in FL1 ovary in comparison to the Ctrl strain. In both gonadal tissues, Agt is downregulated in FL1 compared to Ctrl mice. Transgenic interventional studies already reveal overexpression of the Agt gene in females leads to reduced ovulation capacity (Hefler \& Gregg 2001). Also, antagonizing Ang II type 1 receptor (AGTR1) blocks the growth of dominant follicles and their subsequent rupture, with a simultaneous downregulation of $\mathrm{Ccnd} 2$ mRNA expression. However, in FL1 females, we detected a reduction in Agt expression but upregulation of $C \mathrm{Cnd} 2$ expression. We propose that the altered expression of Agt in the testis is directly linked to the female-focused selection.

Ccnd2 is important in testicular as well as ovarian cell cycle (Lee et al. 2009, Shimizu et al. 2013). Genetic intervention studies report on oligozoospermia and abnormal ovarian folliculogenesis (Sicinski et al. 1996). The Ccnd 2 gene is induced by $\mathrm{FSH}$ and regulates cell proliferation in the gonads (Sicinski et al. 1996). Downregulation by dihydrotestosterone (DHT) induces cell cycle arrest in granulosa cells (Pradeep et al. 2002), while deletion of the gene leads to female sterility (Sicinski et al. 1996).

Furthermore, $H s d 3 b 1$ is a key enzyme in biosynthesis of steroids, which is essential for the production of $\mathrm{P} 4$, which can serve as a precursor of androgens, estrogens and glucocorticoids synthesis. Interestingly, the Hsd3b1 transcript was downregulated in FL1 testis in comparison to Ctrl line. To test for possible physiological effects of this differential $H s d 3 b 1$ expression, we analysed the P4 level in the serum by RIA. The P4 concentrations of the Ctrl males were similar to values found in the literature (Schneider et al. 2003, Deniselle et al. 2016). However, the P4 levels of FL1 bucks were almost 1.5 -fold $(40 \%)$ greater than those of the Ctrl strain. The elevated P4 level in FL1 bucks was surprising because $H s d 3 b 1$ transcripts are decreased in these mice. Although P4 is widely considered a 'female hormone', the serum P4 levels of males do not differ greatly to females in the luteal phase (Zumoff et al. 1990a,b) or post-menopausal women (Oettel \& Mukhopadhyay 2004, Trabert et al. 2015). In men, P4 has been shown to be crucial for spermatogenesis, which is stressed by its induced functional loss ultimately leading to infertility (Abid et al. 2008, Aquila \& De Amicis 2014). The hormone is known to induce the acrosome reaction and to increases the percentage of oocyte penetration (Vigil et al. 2011). Moreover, P4 can trigger hyperactivation in spermatozoa 
due to a rapid increase in $\mathrm{Ca}^{2+}$, possibly mediated by the $\mathrm{GABA}_{A}$ receptor (Kon et al. 2014). Also, the P4 can serve as a precursor for corticosterone, thus feeding the corticosteroid pathway. We previously analysed the serum corticosterone levels in males by gas chromatography coupled to mass spectrometry and detected significantly enhanced (+80\%) corticosterone levels in FL1 compared to Ctrl males (Langhammer et al. 2017). In males, the hormone is known to be either of testicular or adrenal origin.

Furthermore, the transcript of Sult1e1 was downregulated in FL1 testis in comparison to Ctrl strain. Oestrogen (E2) is known to be inactivated by the steroid sulfatase Sult1e1, which is commonly regarded as the only relevant sulfotransferase for estrogens (Geyer et al. 2017). The enzyme Sult1e1 is present in the ovary, whereas its testicular expression and function have not yet been reported (Brown et al. 2006). However, sulfated steroid inactivation, as well as transport and local storage of steroids, have been postulated (Geyer et al. 2017). Also, it could be unfolded that overexpression of Sult1e1 counteracts estrogen-mediated proliferation and decreases the expression of D-cyclin (Xu et al. 2012).

Unexpectedly, we notice an overrepresentation of genes of the haptoglobin-haemoglobin complex in the FL1 testis. We also decocted among the DEGs an enrichment of adaptive immune response, which we currently cannot map to a functional role in reproductive performance. However, this might be rather an indirect effect due to the breeding process and the genetic separation of more than 170 generations causing an undirected allelic drift rather than an active regulation.

\section{Conclusion}

To our knowledge, this is the first study describing global transcriptional changes in the male mice following longterm female-focused, high-fertility selection process, with a still existing and maintained random-mated control mouse line. We detected gene expression changes in the FL1 testis partly associated with female fecundity and ovulation. However, in pig breeding, several races have been selected for different functional traits. Although Landrace and Large White have been selected for high female fertility, the corresponding males do not have superior fertile compared to those breeds selected for other functional traits (e.g. Pietrain, selected for meat quantity and quality) (Kondracki 2003). Thus, our mouse model might serve as a suitable model for pigs or pig breeding strategies. Building on our approach, the FBN 'high-fertility' mouse lines might serve as an informative model to estimate the long-term effects of female-focused breeding on both genders.

\section{Supplementary data}

This is linked to the online version of the paper at https://doi.org/10.1530/REP-17-0392.

\section{Declaration of interest}

The authors declare that there is no conflict of interest that could be perceived as prejudicing the impartiality of the research reported.

\section{Funding}

This work was supported by a grant of the German Research Foundation (DFG) WE2458/10-1.

\section{Author contribution statement}

$M M, A S, J S$ and J M W conceived the experimental design. $M L$ and $N R$ were responsible for animal breeding and line selection. D $\mathrm{K}$ organized RNA preparation, labelling and microarray hybridization. $\mathrm{M} \mathrm{M}$ and $\mathrm{A} S$ conducted the statistical evaluation of microarray data and the qPCR re-analysis, interpreted the results and drafted the manuscript. J M W critically read the manuscript. All authors approved the final manuscript.

\section{Acknowledgements}

The authors thank Ursula Antkewitz, Swanhild Rodewald, Hannelore Klückmann and Petra Reckling for excellent technical assistance. They also acknowledge the staff of the Lab Animal Facility, Leibniz Institute for Farm Animal Biology, for animal care and sample collection.

\section{References}

Abid S, Gokral J, Maitra A, Meherji P, Kadam S, Pires E \& Modi D 2008 Altered expression of progesterone receptors in testis of infertile men. Reproductive BioMedicine Online 17 175-184. (https://doi.org/10.1016/ S1472-6483(10)60192-7)

Alm H, Kuhlmann S, Langhammer M, Tuchscherer A, Torner H \& Reinsch N 2010 Occurrence of polyovular follicles in mouse lines selected for high fecundity. Journal of Reproduction and Development 56 449-453. (https://doi.org/10.1262/jrd.09-224H)

Aquila S \& De Amicis F 2014 Steroid receptors and their ligands: effects on male gamete functions. Experimental Cell Research 328 303-313. (https://doi.org/10.1016/j.yexcr.2014.07.015)

Bader M \& Ganten D 2008 Update on tissue renin-angiotensin systems. Journal of Molecular Medicine 86 615-621. (https://doi.org/10.1007/ s00109-008-0336-0)

Bowman JC \& Falconer DS 1960 Inbreeding depression and heterosis of litter size in mice. Genetics Research 1 262-274. (https://doi. org/10.1017/S0016672300000240)

Brown KA, Dore M, Lussier JG \& Sirois J 2006 Human chorionic gonadotropin-dependent up-regulation of genes responsible for estrogen sulfoconjugation and export in granulosa cells of luteinizing preovulatory follicles. Endocrinology 147 4222-4233. (https://doi. org/10.1210/en.2006-0420)

Brussow KP, Schneider F, Wollenhaupt K \& Tuchscherer A 2011 Endocrine effects of $\mathrm{GnRH}$ agonist application to early pregnant gilts. Journal of Reproduction and Development 57 242-248. (https://doi.org/10.1262/ jrd.10-021O)

Carroll M, Luu T \& Robaire B 2011 Null mutation of the transcription factor inhibitor of DNA binding 3 (id3) affects spermatozoal motility parameters and epididymal gene expression in mice. Biology of Reproduction $\mathbf{8 4}$ 765-774. (https://doi.org/10.1095/biolreprod.110.088344)

Della Beffa C, Cordero F \& Calogero RA 2008 Dissecting an alternative splicing analysis workflow for GeneChip Exon 1.0 ST Affymetrix arrays. BMC Genomics 9 571. (https://doi.org/10.1186/1471-2164-9-571) 
Deniselle MC, Liere P, Pianos A, Meyer M, Aprahamian F, Cambourg A Di Giorgio NP, Schumacher M, De Nicola AF \& Guennoun R 2016 Steroid profiling in male Wobbler mouse, a model of amyotrophic lateral sclerosis. Endocrinology 157 4446-4460. (https://doi.org/10.1210/ en.2016-1244)

Devroey P, Fauser BC, Diedrich K \& Evian Annual Reproduction Workshop G 2009 Approaches to improve the diagnosis and management of infertility. Human Reproduction Update 15 391-408. (https://doi. org/10.1093/humupd/dmp012)

Dietl G, Langhammer M \& Renne U 2004 Model simulations for genetic random drift in the outbred strain Fzt: DU. Archiv Fur Tierzucht-Archives of Animal Breeding 47 595-604. (https://doi.org/10.5194/aab-47-5952004)

Evers JL 2002 Female subfertility. Lancet 360 151-159. (https://doi org/10.1016/S0140-6736(02)09417-5)

Geyer J, Bakhaus K, Bernhardt R, Blaschka C, Dezhkam Y, Fietz D, Grosser G, Hartmann K, Hartmann MF, Neunzig J et al. 2017 The role of sulfated steroid hormones in reproductive processes. Journal of Steroid Biochemistry and Molecular Biology 172 207-221. (https://doi. org/10.1016/j.jsbmb.2016.07.002)

Harris MA, Clark J, Ireland A, Lomax J, Ashburner M, Foulger R, Eilbeck K, Lewis S, Marshall B, Mungall C et al. 2004 The gene ontology (GO) database and informatics resource. Nucleic Acids Research 32 D258D261. (https://doi.org/10.1093/nar/gkh036)

Hefler LA \& Gregg AR 2001 Influence of the angiotensinogen gene on the ovulatory capacity of mice. Fertility and Sterility 75 1206-1211. (https://doi.org/10.1016/S0015-0282(01)01797-6)

Holt M, Vangen O \& Farstad W 2004 Components of litter size in mice after 110 generations of selection. Reproduction 127 587-592. (https://doi.org/10.1530/rep.1.00118)

Kon H, Takei GL, Fujinoki M \& Shinoda M 2014 Suppression of progesterone-enhanced hyperactivation in hamster spermatozoa by gamma-aminobutyric acid. Journal of Reproduction and Development 60 202-209. (https://doi.org/10.1262/jrd.2013-076)

Kondracki S 2003 Breed differences in semen characteristics of boars used in artificial insemination in Poland. Pig News and Information $\mathbf{2 4}$ 119-122.

Kosti I, Jain N, Aran D, Butte AJ \& Sirota M 2016 Cross-tissue analysis of gene and protein expression in normal and cancer tissues. Scientific Reports 6 24799. (https://doi.org/10.1038/srep24799)

Langhammer M, Michaelis M, Hoeflich A, Sobczak A, Schoen J \& Weitzel JM 2014 High-fertility phenotypes: two outbred mouse models exhibit substantially different molecular and physiological strategies warranting improved fertility. Reproduction 147 427-433. (https://doi. org/10.1530/REP-13-0425)

Langhammer M, Michaelis M, Hartmann MF, Wudy SA, Sobczak A, Nurnberg G, Reinsch N, Schon J \& Weitzel JM 2017 Reproductive performance primarily depends on the female genotype in a two-factorial breeding experiment using high-fertility mouse lines. Reproduction 153 361-368. (https://doi.org/10.1530/REP-16-0434)

Lee J, Kanatsu-Shinohara $M$, Morimoto $H$, Kazuki $Y$, Takashima S, Oshimura M, Toyokuni S \& Shinohara T 2009 Genetic reconstruction of mouse spermatogonial stem cell self-renewal in vitro by Ras-cyclin D2 activation. Cell Stem Cell 5 76-86. (https://doi.org/10.1016/j. stem.2009.04.020)

Maruta H \& Arakawa K 1983 Confirmation of direct angiotensin formation by kallikrein. Biochemical Journal 213 193-200. (https://doi. org/10.1042/bj2130193)

Matzuk MM \& Lamb DJ 2008 The biology of infertility: research advances and clinical challenges. Nature Medicine 14 1197-1213. (https://doi. org/10.1038/nm.f.1895)

Mi H, Lazareva-Ulitsky B, Loo R, Kejariwal A, Vandergriff J, Rabkin S, Guo N, Muruganujan A, Doremieux O, Campbell MJ et al. 2005 The PANTHER database of protein families, subfamilies, functions and pathways. Nucleic Acids Research 33 D284-D288. (https://doi. org/10.1093/nar/gki078)

Michaelis M, Langhammer M, Hoeflich A, Reinsch N, Schoen I \& Weitzel JM 2013 Initial characterization of an outbreed mouse model for male factor (in)fertility. Andrology 1 772-778. (https://doi.org/10.1111/ j.2047-2927.2013.00108.x)

Oettel M \& Mukhopadhyay AK 2004 Progesterone: the forgotten hormone in men? Aging Male 7 236-257. (https://doi org/10.1080/13685530400004199)
Pfaffl MW, Horgan GW \& Dempfle L 2002 Relative expression software tool (REST) for group-wise comparison and statistical analysis of relative expression results in real-time PCR. Nucleic Acids Research 30 e36. (https://doi.org/10.1093/nar/30.9.e36)

Pradeep PK, Li X, Peegel H \& Menon KM 2002 Dihydrotestosterone inhibits granulosa cell proliferation by decreasing the cyclin D2 mRNA expression and cell cycle arrest at G1 phase. Endocrinology 143 2930-2935. (https://doi.org/10.1210/endo.143.8.8961)

Schneider JS, Stone MK, Wynne-Edwards KE, Horton TH, Lydon J, O'Malley B \& Levine JE 2003 Progesterone receptors mediate male aggression toward infants. PNAS 100 2951-2956. (https://doi. org/10.1073/pnas.0130100100)

Schüler L BL 1982 Die reproduktive Lebensleistung auf Fruchtbarkeit selektierter Labormauslinien. Archiv Fur Tierzucht-Archives of Animal Breeding 25 275-281.

Shimizu T, Hirai Y \& Miyamoto A 2013 Expression of cyclins and cyclin-dependent kinase inhibitors in granulosa cells from bovine ovary. Reproduction in Domestic Animals 48 e65-e69. (https://doi. org/10.1111/rda.12177)

Sicinski P, Donaher JL, Geng Y, Parker SB, Gardner H, Park MY, Robker RL, Richards JS, McGinnis LK, Biggers JD et al. 1996 Cyclin D2 is an FSHresponsive gene involved in gonadal cell proliferation and oncogenesis. Nature 384 470-474. (https://doi.org/10.1038/384470a0)

Spitschak M, Langhammer M, Schneider F, Renne U \& Vanselow J 2007 Two high-fertility mouse lines show differences in component fertility traits after long-term selection. Reproduction, Fertility, and Development 19 815-821. (https://doi.org/10.1071/RD07009)

Suto J 2015 Genetic analysis of litter size in mice. Journal of Veterinary Medical Science 77 353-358. (https://doi.org/10.1292/jvms.14-0350)

Trabert B, Falk RT, Stanczyk FZ, McGlynn KA, Brinton LA \& Xu X 2015 Reproducibility of an assay to measure serum progesterone metabolites that may be related to breast cancer risk using liquid chromatography-tandem mass spectrometry. Hormone Molecular Biology and Clinical Investigation 23 79-84. (https://doi.org/10.1515/ hmbci-2015-0026)

Vanselow J, Nurnberg G, Koczan D, Langhammer M, Thiesen HJ \& Reinsch N 2008 Expression profiling of a high-fertility mouse line by microarray analysis and qPCR. BMC Genomics 9 307. (https://doi. org/10.1186/1471-2164-9-307)

Vigil P, Orellana RF \& Cortes ME 2011 Modulation of spermatozoon acrosome reaction. Biological Research 44 151-159. (https://doi. org/10.4067/S0716-97602011000200007)

Williams B 2016 Drug discovery in renin-angiotensin system intervention: past and future. Therapeutic Advances in Cardiovascular Disease 10 118-125. (https://doi.org/10.1177/1753944716642680)

Xu Y, Liu X, Guo F, Ning Y, Zhi X, Wang X, Chen S, Yin L \& Li X 2012 Effect of estrogen sulfation by SULT1E1 and PAPSS on the development of estrogen-dependent cancers. Cancer Science 103 1000-1009. (https://doi.org/10.1111/j.1349-7006.2012.02258.x)

Ye J, Coulouris G, Zaretskaya I, Cutcutache I, Rozen S \& Madden TL 2012 Primer-BLAST: a tool to design target-specific primers for polymerase chain reaction. BMC Bioinformatics 13 134. (https://doi. org/10.1186/1471-2105-13-134)

Zumoff B, Miller L, Levin J, Levit CD, Miller EH, Heinz U, Kalin M, Denman H, Jandorek R \& Rosenfeld RS 1990a Follicular-phase serum progesterone levels of nonsmoking women do not differ from the levels of nonsmoking men. Steroids 55 557-559. (https://doi.org/10.1016/0039128X(90)90052-D)

Zumoff B, Miller L, Poretsky L, Levit CD, Miller EH, Heinz U, Denman H, Jandorek R \& Rosenfeld RS 1990b Subnormal follicular-phase serum progesterone levels and elevated follicular-phase serum estradiol levels in young women with insulin-dependent diabetes. Steroids 55 560-564. (https://doi.org/10.1016/0039-128X(90)90053-E)

Received 28 June 2017

First decision 14 August 2017

Revised manuscript received 2 November 2017

Accepted 1 December 2017 